\title{
ENSINO SOBRE CUIDADO INTERDISCIPLINAR ATRAVÉS DE SIMULAÇÃO: UMA PROPOSTA METODOLÓGICA
}

Gabriele da Silva Reis Pessoa, Kátia Cristina Cândido de Oliveira Santos, Elaine Cristina Negri Santos, Eduardo Fuzetto Cazañas.

Universidade do Oeste Paulista - UNOESTE, Faculdade de Enfermagem, Presidente Prudente, SP. E-mail: eduardocazanas@unoeste.br

\section{RESUMO}

O objetivo deste estudo foi analisar a satisfação dos residentes quanto à formação interdisciplinar a partir do Laboratório de Prática Simulada. Trata-se de um estudo de campo com abordagem quantitativa. Os dados foram coletados numa universidade do Oeste Paulista e envolveu 12 residentes multiprofissionais, com aprovação do Comitê de Ética parecer no 1.508.897. Verificouse alto grau de satisfação dos residentes quanto promoção de práticas e discussões interdisciplinares $O$ Laboratório de Prática Simulada revelou-se como uma estratégia de ensino interdisciplinar, assim, pode ser experimentada pelos cursos de graduação em saúde da universidade em questão. Como limitação, cita-se o pequeno número de sujeitos da pesquisa demandando maiores estudos.

Palavras chave: Enfermagem, Residência Internato não médico, Simulação de Paciente, Ensino, Integralidade em Saúde.

\section{INTERDISCIPLINARY CARE THROUGH SIMULATION TEACHING: a methodological proposal}

\begin{abstract}
The objective of this study was to analyze the residents satisfaction regarding the interdisciplinary training from the Simulated Practice Laboratory. This is a field study with a quantitative approach. Data were collected at a university in the West region of São Paulo and involved 12 multiprofessional residents, with the approval of the Ethics Committee opinion $\mathrm{n} 01,508,897$. There was a high degree of satisfaction among residents regarding the promotion of interdisciplinary practices and discussions. The Simulated Practice Laboratory turned out to be an interdisciplinary teaching strategy, so it can be tried out by undergraduate health courses at the university in question. As a limitation, we cite the small number of subjects requiring more studies. Keyword: Nursing, Internship Nonmedical, Patient Simulation, Teaching, Integrality in Heath
\end{abstract}




\section{INTRODUÇÃO}

O Sistema Único de Saúde (SUS) é um dos serviços de saúde pública mais complexos do mundo, tendo em vista os desafios para alcançar um dos seus princípios, a integralidade. Neste sentido o Ministério da Educação e Saúde propõem em 2005 a Residência Multiprofissional em Saúde, privilegiando a Atenção Básica $(A B)$ e processos de formação com característica interdisciplinar, crítico e reflexiva e que proporcionem autonomia progressiva aos residentes. ${ }^{(1-5)}$

Consciente da necessidade de aperfeiçoar práticas profissionais numa lógica que vá ao encontro do trabalho interdisciplinar e na perspectiva da integralidade, uma Universidade do Interior do Estado de São Paulo em parceria com um Hospital Estadual iniciaram a primeira turma de Residentes Multiprofissionais em março de $2014 .^{6}$

Entre as profissões que compõem residentes e professores, destaca-se a enfermagem, que propôs a residência, a utilização de um método de simulação em desenvolvimento no curso desde 2013. Trata-se do ensino baseado em simulação, que proporciona aprendizagem significativa, crítico e reflexiva num ambiente seguro. ${ }^{(7-9)}$

O ensino por simulação pode ser utilizado de diversas formas, desde para uma prática "simples" de punção venosa em uma peça de manequim sem contextualização com a realidade; um atendimento de alta complexidade com manequim "robô" ou um paciente real num contexto que abarca além de questões clínicas, as questões sócio econômicas, de acesso aos serviços de saúde e autonomia. Podem ser realizados ainda dentro do aspecto pedagógico tradicional/tecnicista, ou associados metodologias ativas de ensino-aprendizagem. ${ }^{\text {(10-11-12-13-14-15) }}$

$\mathrm{Na}$ intenção de promover uma metodologia de ensino coerente com as reais necessidades de saúde da população atendidas pelo (SUS), foi proposto os residentes a proposta de ensino e aprendizagem denominado Laboratório de Prática Simulada(LPS), que foi baseado no Laboratório de Prática Profissional, criado em uma faculdade pública do estado de São Paulo.

Por tratar-se de uma nova proposta de metodologia de ensino em simulação e por ser a primeira aplicação de cunho interprofissional, faz-se necessário verificar a satisfação dos residentes em relação ao potencial do LPS no ensino interdisciplinar em três cenários peculiares.

Neste sentido foi elaborada a seguinte questão de pesquisa: "Qual é a capacidade do LPS promover práticas/discussões interdisciplinares?"

Desta forma, o objetivo desta pesquisa é analisar a satisfação dos residentes quanto a capacidade do LPS produzir práticas e discussões interdisciplinares.

\section{METODOLOGIA}

Trata-se de uma pesquisa de abordagem quantitativa de modelo não experimental, descritivo e de corte transversal. Foram os sujeitos da pesquisa: 12 residentes (100\%), matriculados no $2^{\circ}$ ano da Residência Multiprofissional do Idoso, Intensivismo e Urgência/trauma, sendo três enfermeiros, três farmacêuticos, três fisioterapeutas e três nutricionistas.

Os participantes da pesquisa receberam todas as informações necessárias quanto ao desenvolvimento da pesquisa, antes, durante e após sua realização, ficando cientes de que a participação ocorrerá de acordo com as suas vontades, estando livres para desistência.

O Laboratório de Prática Simulada ocorreu na Casa Simulada e Laboratório de Habilidades e Simulação da Universidade do Oeste Paulista, ${ }^{16,18}$ em três ciclos de aprendizagem, sendo que cada ciclo foi composto por síncrese (simulação, avaliação 360 , problematização e levantamento de questões de aprendizagem), análise (momento de busca individual qualificada por meio de artigos, livros, vídeos e consultoria) e síntese (propostas de resolução dos problemas/questões com embasamento teórico).

Para elaboração das situações simuladas e mediação das discussões o autor estava embasado na Pedagogia Histórico Crítica proposta por Saviani ${ }^{14}$, Metodologia da Problematização 
proposta por Berbel, ${ }^{19}$ Taxonomia das Necessidades de Saúde de Cecílio e Matsumoto ${ }^{20}$ e Grupo Operativo proposto por Pichon Riviere. ${ }^{21}$

Foram trabalhadas três simulações utilizando a estratégia de simulação híbrida com Paciente Estandarizado e manequins de média fidelidade.

As Simulações se deram em torno da história de "Antônia", uma jovem portadora de Linfoma de Hodgkins que veio do nordeste em busca de maior acesso a tecnologias de diagnóstico e tratamento. Ocorreram em três contextos: $A B$, na qual a paciente passa por atendimento em um consultório simulado da Estratégia Saúde da Família; no Hospital, onde passa por atendimento na Clínica Médica, após o agravamento do seu quadro e por fim, em uma Visita Domiciliária, onde os profissionais deparam-se com a fase terminal da doença.

Após a aprovação do Comitê de Ética (53455416.0.0000.5515) e assinatura do Termo de Consentimento Livre e Esclarecido a coleta de dados foi realizada ao final de cada encontro entre os meses de maio a junho de 2016, através do preenchimento de escala Phrase Completion, com variáveis indicando o grau de satisfação dos residentes com o LPS.

Phrase Completion trata-se de uma escala padrão de 11 pontos de $0-10$, onde 0 é associado a ausência de atributo e 10 tem relação com a intensidade máxima de sua presença. Foi desenvolvida por Hofge e Gillespie ${ }^{22}$ como alternativa a escala de Likert.

Os dados foram codificados em planilha eletrônica Microsoft Office Excel $^{\circledR}$ versão 2010 e realizado análise de frequência simples relativa com auxílio do software $\mathrm{R} 1$, tendo sido utilizado o teste $\mathrm{G}$, ao nível de significância de $5 \%{ }^{23}$

\section{RESULTADOS}

Perfil dos Sujeitos Investigados

As 12 residentes são todas do sexo feminino, com idade mínima de 23 anos, máxima de 35 sendo média 25 anos. Destas, 41,7\% graduaram-se no ano de 2014, 33,3\% em 2013 e 25\% em 2010. Duas $(16,7 \%)$ possuem além da formação em saúde, graduação em Bioquímica e História e nenhuma (\%) entre possuem curso de pós-graduação.

Tabela 1. Distribuição percentual quanto à satisfação relacionada ao LPS promover práticas e discussões interdisciplinares, Presidente Prudente, 2016.

\begin{tabular}{llllllll}
\hline & \multicolumn{2}{l}{ 1․ Ciclo } & \multicolumn{2}{l}{ 2․ Ciclo } & \multicolumn{2}{l}{ 3․ Ciclo } & p-valor \\
\hline Satisfação & $\mathrm{n}$ & $\%$ & $\mathrm{n}$ & $\%$ & $\mathrm{n}$ & $\%$ & \\
\hline Muito pequena & 0 & 0 & 0 & 0 & 0 & 0 & \\
Moderada & 0 & 0 & 0 & 0 & 0 & 0 & 0,6157 \\
Muito grande & 11 & 92,0 & 12 & 100,0 & 11 & 92,0 & \\
Não respondeu & 1 & 8,0 & 0 & 0 & 1 & 8,0 & \\
\hline Total & 12 & 100,0 & 12 & 100,0 & 12 & 100,0 & \\
\hline
\end{tabular}

Nesta tabela, que no 1ㅇ e 3 ciclos $92 \%$ dos residentes atribuíram o conceito Muito Grande e no 2 ciclo $100 \%$ atribuíram o mesmo valor em relação ao LPS promover práticas e discussões interdisciplinares. Desta forma, observa-se homogeneidade dos dados e o potencial em proporcionar um ensino com cunho interdisciplinar.

\section{DISCUSSÃO}

Novas propostas de metodologias de Ensino Baseada em Simulação são possíveis de serem experimentadas, em detrimento à flexibilização dos currículos propostos pelas Diretrizes Curriculares Nacionais e no sentido de aprimorar o processo de Ensino que vá ao encontro dos princípios e diretrizes do SUS. ${ }^{13}$ 
O enfoque interdisciplinar e a lógica do trabalho em equipes multiprofissionais, raramente são explorados pelas IES na graduação, o que se reproduz nas equipes de saúde, resultando em ações isoladas de cada profissional e na sobreposição das práticas de cuidado e sua fragmentação. ${ }^{4,13,24}$

Neste sentido, o ensino atrelado à interdisciplinaridade é essencial para que sejam desenvolvidas competência profissional, que sejam condizentes com as necessidades das pessoas, assim como os princípios e diretrizes que permeiam o SUS. ${ }^{4}$

\section{CONCLUSÃO}

O ato de aprender é um processo contínuo devendo ser parte reflexiva para o aperfeiçoamento dentro das vastas possibilidades pedagógicas. O LPS demonstrou-se uma estratégia de ensino e aprendizagem que pode ser utilizada para além do curso de enfermagem, visto seu caráter interdisciplinar.

Neste sentido, ofertar-se-á de forma experimental o LPS como estratégia de ensino a outros cursos de graduação da universidade em que foi realizada a pesquisa.

Como limitação cita-se o pequeno número de sujeitos da pesquisa, no entanto, novos estudos com caráter quanti/qualitativo estão sendo desenvolvidos.

\section{REFERÊNCIAS}

1. Lima, M.; Reibnitz, K. S.; Prado, M. L. do and Kloh, Daiana. Integralidade como princípio pedagógico na formação do enfermeiro. Texto contexto - enferm. [online]. 2013, vol.22, n.1, pp.106-113. ISSN 0104-0707. http://dx.doi.org/10.1590/S0104-07072013000100013.

2. Mendes, E.V. O cuidado das condições crônicas da Atenção Primária à Saúde: o imperativo das consolidação da Estratégia Saúde da Família. Brasília (DF): Organização Pan-Americana de Saúde, 2012.

Disponível em:

http://bvsms.saude.gov.br/bvs/publicacoes/cuidado_condicoes_atencao_primaria_saude.pdf>. Acesso em: 06 ago. 2015.

3. Brasil. Ministério da Saúde. Secretaria de Gestão do Trabalho e da Educação na Saúde. Departamento de Gestão da Educação na Saúde. Residência multiprofissional em saúde: experiências, avanços e desafios / Ministério da Saúde, Secretaria de Gestão do Trabalho e da Educação na Saúde, Departamento de Gestão da Educação em Saúde. Brasília: Ministério da Saúde, 2006. ISBN 85-334-1298-3

4. Winters, J. R. F; Do Prado, M. L; Heidemann, I. T. S. B.: A formação em enfermagem orientada aos princípios do Sistema Único de Saúde: percepção dos formandos. Escola Anna Nery, v. 20, n. 2, p. 248-253, 2016.

5. Fazenda, I.C.A (org). O que é interdisciplinaridade? São Paulo (SP): Cortez, 2008.

6. Unoeste. Residência em saúde do idoso oferta bolsas de $\mathrm{R} \$ 2,9$ mil, presidente prudente-sp, 24 de jan. 2014 Disponível em:<http://www.unoeste.br/site/noticias/2014/1/residencia-em-saudedo-idoso-oferta-bolsas-de-r-29-mil.html>.Acesso em 14 de novembro de 2015.

7. Costa, N.L.; Cazañas, E.F. Relação interdisciplinar na formação do farmacêutico. In: Encontro de Ensino, Pesquisa e Extensão: Anais eletrônicos. Presidente Prudente, 2014. Disponível em: <https://www.unoeste.br/site/enepe/2014/Anais/CienciasSaude/Farm\%C3\%A1cia.pdf>. Acesso em 06 ago 2015. 
8. Milhorança AF, Sanvezzo, VMS, Cazañas EF. Simulação como metodologia de ensino e avaliação na perspectiva da integralidade. In: Anais do 21ㅇ Anais do Encontro de Ensino, Pesquisa e Extensão, 2015; Presidente Prudente. Presidente Prudente: Universidade do Oeste Paulista; 2015. $\mathrm{p}$ 455. [acesso em 2016 out 23]. Disponível em: https://www.unoeste.br/site/enepe/2015/Anais/CienciasSaude/Enfermagem.pdf

09. Waterkemper, Roberta; Do Prado, Marta Lenise. Estratégias de ensino-aprendizagem em cursos de graduação em Enfermagem. av.enferm. [online]. 2011, vol.29, n.2, pp.234-246. ISSN 0121-4500.

10. Brandão, HP et al. Gestão de desempenho por competências: integrando a gestão por competências, o balanced scorecard e a avaliação 360 graus. Revista de Administração Pública RAP, RIO DE JANEIRO 42(5):875-98, SET/OUT. 2008. https://doi.org/10.1590/S0034$\underline{76122008000500004}$

11. Barreto, G.G. Simulação realística como estratégia de ensino para o curso de graduação em enfermagem: revisão integrativa. Revista Baiana de Enfermagem, Salvador, 28 (2): 208-214 maio/ago. 2014

12. Hafner, M.L.M.G et al. A formação médica e a clínica ampliada: resultados de uma experiência brasileira. Ciência \& Saúde Coletiva, 15(Supl. 1):1715-1724, 2010. https://doi.org/10.1590/S1413$\underline{81232010000700083}$

13. Moraes, M.A.A et. al. O uso da simulação como estratégia avaliativa na graduação em saúde. In: Avaliação nos cursos de Medicina e Enfermagem: perspectivas e desafios. Moraes Curitiba: CRV, 2012.

14. Saviani D. Escola e democracia. 41a ed. Campinas (SP): Autores Associados; 2009.

15. Braccialli, LAD et al. Avaliação do estudante: no exercício de avaliação da prática profissional. Avaliação, Campinas; Sorocaba (SP). 2008; 13 (1): 101-18. https://doi.org/10.1590/S1414$\underline{40772008000100006}$

16. Universidade do Oeste Paulista (UNOESTE). Sobre o Laboratório de Habilidades e Simulação [Internet]. Presidente Prudente; 2016. [citado 06 nov 2016]. Disponível em: http://www.unoeste.br/Ihabsim/historia.php

17. Franco, C.A.G.S et al . OSCE para Competências de Comunicação Clínica e Profissionalismo: Relato de Experiência e Meta-Avaliação. Rev. bras. educ. med., Rio de Janeiro , v. 39, n. 3, p. 433441, Sept. 2015 Disponível em: <http://www.scielo.br/scielo.php?script=sci_arttext\&pid=S010055022015000300433\&lng=en\&nrm=iso>. Acesso em 06 nov. 2016.

18. Universidade do Oeste Paulista (UNOESTE). Casa Simulada no Campus I é inaugurada pela Enfermagem. [Internet]. Presidente Prudente; 2016. [citado 06 nov 2016]. Disponível em: https://www.unoeste.br/Noticias/2015/11/casa-simulada-no-campus-i-e-inaugurada-pelaenfermagem 
19. Berbel NAN. A metodologia da problematização com o Arco de Maguerez: uma reflexão teórico-epistemológica. Londrina (PR): EDUEL; 2012.

20. Cecílio, LCO, Matsumoto, NF. Uma taxonomia operacional de necessidades de saúde.

In: PINHEIRO R; FERLA A F; MATTOS R A (orgs.). Gestão em Redes: tecendo os fios da integralidade em saúde. Rio Grande do Sul: Rio de Janeiro: EdUCS/UFRS: IMS/UERJ: CEPESC, 2006. 112p. ISBN: 85-89737-29-2

21. Pichon, R.E. O processo grupal. 8a ed. São Paulo: Martins Fontes ,2009.

22. Hodge, D. R.; Gillespie, D. F. Phrase completion: an alternative to Likert scales. Social Work Research, 27 (1), p. 45-55, 2003. https://doi.org/10.1093/swr/27.1.45

23. R. Core Team. A language and environment for statistical computing. R. Foundation for Statistical Computing Vienna, Austria, 2016.

24. Overeem, K; Wollersheim, HC; Arah, JK; Grol, RPTM; Lombarts, KMJMH. Evaluation of physicians' professional performance: An iterative development and validation study of multisource feedback instruments. BMC Health Services Research 2012. [citado 06 nov. 2016]: 12(80): 2-11. Disponível em: http://www. biomedcentral.com/1472-6963/12/80 